\title{
GPS IIR-M L1 Transmit Power Redistribution: Analysis of GNSS Receiver and High-Gain Antenna Data
}

\author{
Steffen Thoelert, German Aerospace Center (DLR), Institute for Communications and Navigation (IKN), \\ Oberpfaffenhofen, Germany
}

André Hauschild, Peter Steigenberger, German Aerospace Center (DLR), German Space Operations Center (GSOC), Oberpfaffenhofen, Germany

Richard B. Langley, Department of Geodesy and Geomatics Engineering, University of New Brunswick, Fredericton, NB, Canada

Felix Antreich, Department of Teleinformatics Engineering, Federal University of Ceará (UFC), Fortaleza, Brazil

\begin{abstract}
All seven operational GPS Block IIR-M satellites were subject to short maintenance periods between February 7 and 8, 2017. With the help of data from several tracking stations of the International GNSS Service (IGS) and high-gain antenna measurements, it could be identified that the satellites' transmit power of different L1 signal components has been changed during the maintenance. The analysis shows that the total radiated power of the satellites remains constant, but the power of the C/A-code and the $\mathrm{P}(\mathrm{Y})$-code signals are increased whereas the powers of the $\mathrm{M}$-code and the intermodulation product are reduced. A detailed look reveals a more efficient use of the total available power on the spacecraft through the decreased power of the intermodulation product. Thus, in sum more power is available for the three navigation signals on L1.

Observations from geodetic GNSS receivers were analyzed to demonstrate the effect of the change in L1 signal power distribution on the measured $\mathrm{C} / \mathrm{N}_{0}$ of the $\mathrm{C} / \mathrm{A}$-code and $\mathrm{P}(\mathrm{Y})$-code for different receiver types. High-gain antenna data collected before and after the maintenance periods are used to analyze improved utilization of the available power through the reduction of the losses caused by the intermodulation product.
\end{abstract}

\section{INTRODUCTION}

Between February 7, 05:02 UTC and February 8, 12:30 UTC of 2017 all operational GPS Block IIR-M satellites were consecutively unavailable for short periods of time. The GPS constellation comprises in total eight Block IIR-M satellites of which seven are fully operational. This satellite type is a modernized version of the Block IIR type and was launched between 2005 and 2009. In addition to the legacy GPS L1 C/A-code and L1/L2 P(Y)-code signals, it also provides an additional military signal, the M-code on L1 and L2, and a new civil signal, the L2Ccode on L2. The military M-code signal uses a sine binary offset carrier modulation with a sub-carrier frequency of $10.23 \mathrm{MHz}$ and a chip rate of $5.115 \mathrm{Mcps}\left(\mathrm{BOC}_{\mathrm{sIN}}(10,5)\right)$ and cannot be tracked by civilian receivers since the necessary signal properties are not publicly available. The civil L2C-code consists of time-multiplexed data and pilot components, resulting in a binary phase-shift keying modulation with chip rate of 1.023 Mcps (BPSK(1)) (IS-GPS-200H 2014, Hartman et al. 2000, Rajan and Tracy 2003). One of the Block IIR-M satellites has been equipped with an experimental signal generation unit to broadcast another civil signal on the third GPS frequency 
L5. This satellite has never been set usable due to internal signal reflection caused by the additional instrument (Ericson et al. 2010).

The maintenance periods were announced ahead of time through seven Notice Advisories to Navstar Users (NANUs). The first satellite to become unavailable was PRN17 (SVN 53). Its maintenance period lasted for 54 minutes. The other six satellites were affected by shorter maintenance periods of only 21 minutes and 29 minutes. An earlier NANU no. 005 released on January 19, 2017, states that "The 2d Space Operations Squadron (2 SOPS) periodically conducts configuration changes on GPS satellites to assess current capabilities, validate future capabilities and ensure continued interoperability [...]”. In addition, the Civil GPS Service Interface Committee Executive Secretariat published a statement on January 25, 2017: "Beginning 25 January 2017, Air Force Space Command (AFSPC) will conduct a limited duration test implementing an increase of the L1 C/A power level on the GPS Block IIR-M and IIF satellites (19 vehicles) [...]”. The high frequency of satellite maintenance periods with short duration as happened in early February is uncommon and thus a connection to these announcements was quickly suspected and investigated through an analysis of measured carrier-to-noisedensity ratios $\left(\mathrm{C} / \mathrm{N}_{0}\right)$ of geodetic receivers and high-gain antenna measurements.

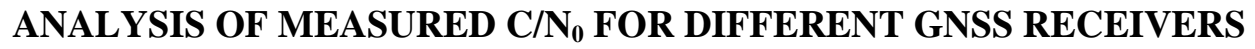

The most obvious effect of the satellite reconfiguration during the unhealthy periods was a change in the measured $\mathrm{C} / \mathrm{N}_{0}$ of the $\mathrm{C} / \mathrm{A}$-code signal as reported by geodetic receivers. Analysis of the data of several stations of the International GNSS Service (IGS; Dow et al., 2009) indicates that the satellites' transmit power for this signal was increased. The plots in Figure 1 and Figure 2 depict the $C / \mathrm{N}_{0}$ over time for satellite passes on three consecutive days (February 6-8, 2017). Figure 1 shows the results for PRN 12 tracked by a Septentrio PolaRx4TR receiver (USN8) located in Washington, DC, USA. The pass during which the maintenance occurs is plotted in magenta. The unhealthy period is indicted by the gray shaded area. The receiver obviously does not track unhealthy satellites, since no observations are available during that time despite the transmitter being continuously on. A noticeable increase in the $\mathrm{C} / \mathrm{N}_{0}$ from about $50 \mathrm{~dB}-\mathrm{Hz}$ to approximately $52 \mathrm{~dB}-\mathrm{Hz}$ is visible after the tracking outage. The $\mathrm{C} / \mathrm{N}_{0}$ for February 8 (plotted in blue) is clearly shifted toward larger values compared to February 6, which indicates that the satellite continued to transmit with increased power. The receiver does not provide $\mathrm{L} 1 \mathrm{P}(\mathrm{Y})$-code measurements and no change could be detected for the $\mathrm{C} / \mathrm{N}_{0}$ of the $\mathrm{L} 2$ $\mathrm{P}(\mathrm{Y})$-code.

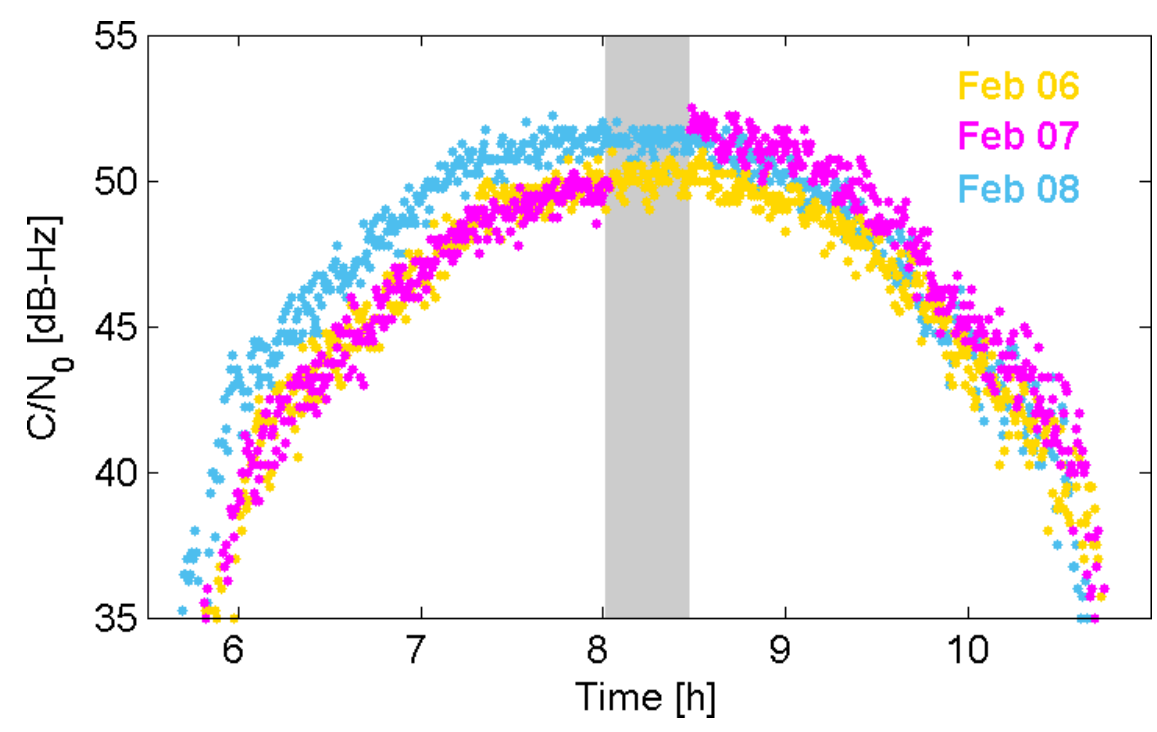

Figure 1: Plot of L1 C/A C/ $\mathrm{N}_{0}$ over time (UTC) for consecutive satellite passes of PRN 12 (SVN 58) tracked by a Septentrio PolaRx4TR receiver located in Washington, DC, USA, on February 6-8, 2017. The satellite's unhealthy period on February 7 is indicated by the gray shaded area. 
The same analysis has been done for PRN 5 and observations from a Septentrio PolaRx4 receiver at the IGS station KOUR located in Kourou, French Guiana. The results are depicted in Figure 2. This receiver tracks the satellite during the unhealthy period. A distinct step in $\mathrm{C} / \mathrm{N}_{0}$ is clearly visible shortly after the satellite switches to unhealthy. The power increase is also permanent on this satellite, as it continues to transmit with higher power on the following day. The same observations as in Figure 1 and Figure 2 can also be made for the other operational Block IIR-M satellites and other receivers. A repeated analysis for the Block IIR-M satellites on February 22 confirmed that the L1 C/A power levels were still increased.

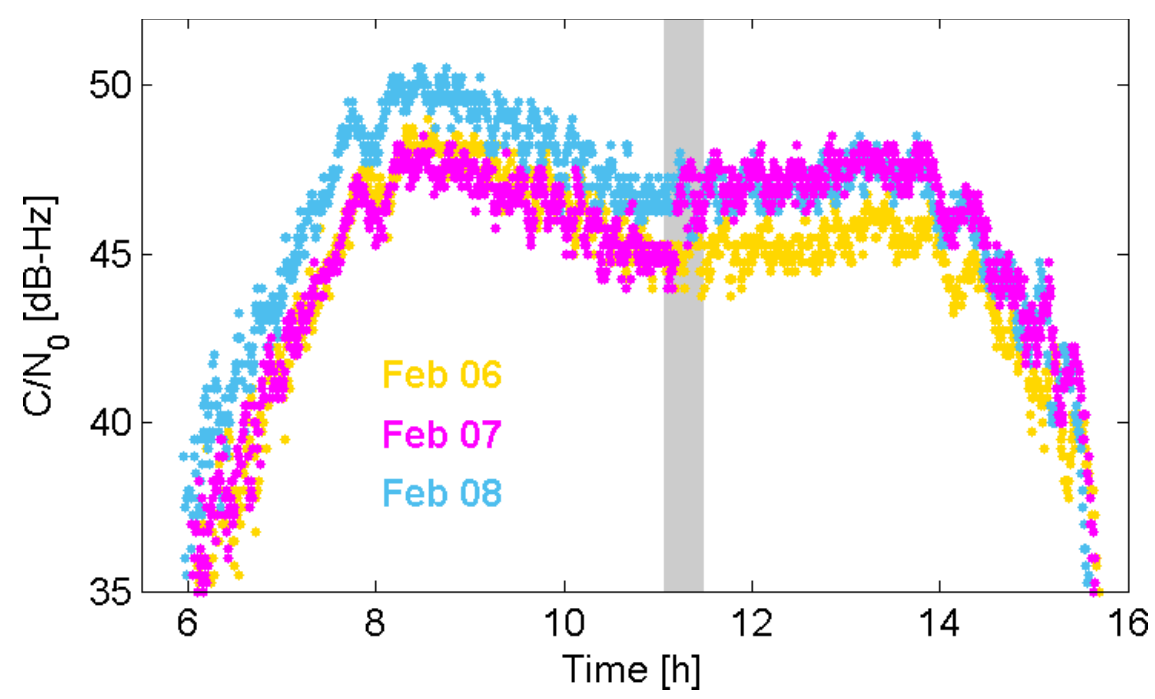

Figure 2: Plot of L1 C/A C/ $\mathrm{N}_{0}$ over time (UTC) for consecutive satellite passes of PRN 5 (SVN 50) tracked by a Septentrio PolaRx4 receiver located in Kourou, French Guiana, on February 6-8, 2017. The satellite's unhealthy period on February 7 is indicated by the gray shaded area.

The difference between the measured $\mathrm{C} / \mathrm{N}_{0}$ before and after the unhealthy period is typically $1-2 \mathrm{~dB}$. On average, the measured $\mathrm{C} / \mathrm{N}_{0}$ is $1.5 \mathrm{~dB}$ higher with a scatter of $\pm 0.25 \mathrm{~dB}$ among the various satellites. Further analysis reveals that different receivers respond with a different change in $\mathrm{C} / \mathrm{A}$-code $\mathrm{C} / \mathrm{N}_{0}$ to the transmit power change. Table 1 summarizes the results for Leica, NovAtel, Septentrio and Trimble receivers, which report $\mathrm{C} / \mathrm{N}_{0}$ changes between $1 \mathrm{~dB}$ and $2 \mathrm{~dB}$. Manufacturer-specific algorithms for $\mathrm{C} / \mathrm{N}_{0}$ estimation obviously hamper the usefulness of measured $\mathrm{C} / \mathrm{N}_{0}$ as a precise indicator of received signal power strength.

Table 1: Changes in $\mathrm{L} 1 \mathrm{C} / \mathrm{A}$-code $\mathrm{C} / \mathrm{N}_{0}[\mathrm{~dB}]$ for different receiver types obtained from differences for days before and after the increase of the transmit power.

\begin{tabular}{|l|l|c|c|c|c|c|c|c|}
\hline \multirow{2}{*}{ Receiver } & \multirow{2}{*}{ Station(s) } & \multicolumn{7}{|c|}{ PRN/SVN } \\
\cline { 3 - 9 } & & $\begin{array}{c}\text { PRN 17 } \\
\text { SVN 53 }\end{array}$ & $\begin{array}{c}\text { PRN 12 } \\
\text { SVN 58 }\end{array}$ & $\begin{array}{c}\text { PRN 5 } \\
\text { SVN 50 }\end{array}$ & $\begin{array}{c}\text { PRN 15 } \\
\text { SVN 55 }\end{array}$ & $\begin{array}{c}\text { PRN 31 } \\
\text { SVN 52 }\end{array}$ & $\begin{array}{c}\text { PRN 7 } \\
\text { SVN 48 }\end{array}$ & $\begin{array}{c}\text { PRN 29 } \\
\text { SVN 57 }\end{array}$ \\
\hline Leica & KOUG & 1.4 & 1.4 & 1.3 & 1.3 & 1.2 & 1.6 & 1.6 \\
\hline NovAtel & USN9 & 0.9 & 1.1 & 1.0 & 1.1 & 1.0 & 1.3 & 1.6 \\
\hline Septentrio & KOUR, USN8, YEL2 & 1.6 & 1.6 & 1.7 & 1.8 & 1.6 & 2.1 & 2.1 \\
\hline Trimble & LMMF, PFRR, UCAL & 1.9 & 1.8 & 1.9 & 2.0 & 1.7 & 2.2 & 2.2 \\
\hline
\end{tabular}

\section{HIGH-GAIN ANTENNA MEASUREMENTS}

As an independent confirmation of the transmit power change on the GPS Block IIR-M satellites, measurements with DLR's $30 \mathrm{~m}$ high-gain antenna at Weilheim have been recorded. Figure 3 depicts the L1 spectral flux density for December 7, 2015 and for March 4, 2017 for PRN 29 captured at same elevation to provide comparability. 
The sharp peak in the middle of the spectrum represents the C/A-code. Comparing the measurements from March 2017 to December 2015 makes obvious that the power has been increased. The plot also shows that the M-code flux density with main lobes near 1565 and $1585 \mathrm{MHz}$ is reduced in March 2017 compared to December 2015. The $\mathrm{P}(\mathrm{Y})$ signal strength remains essentially unaltered.

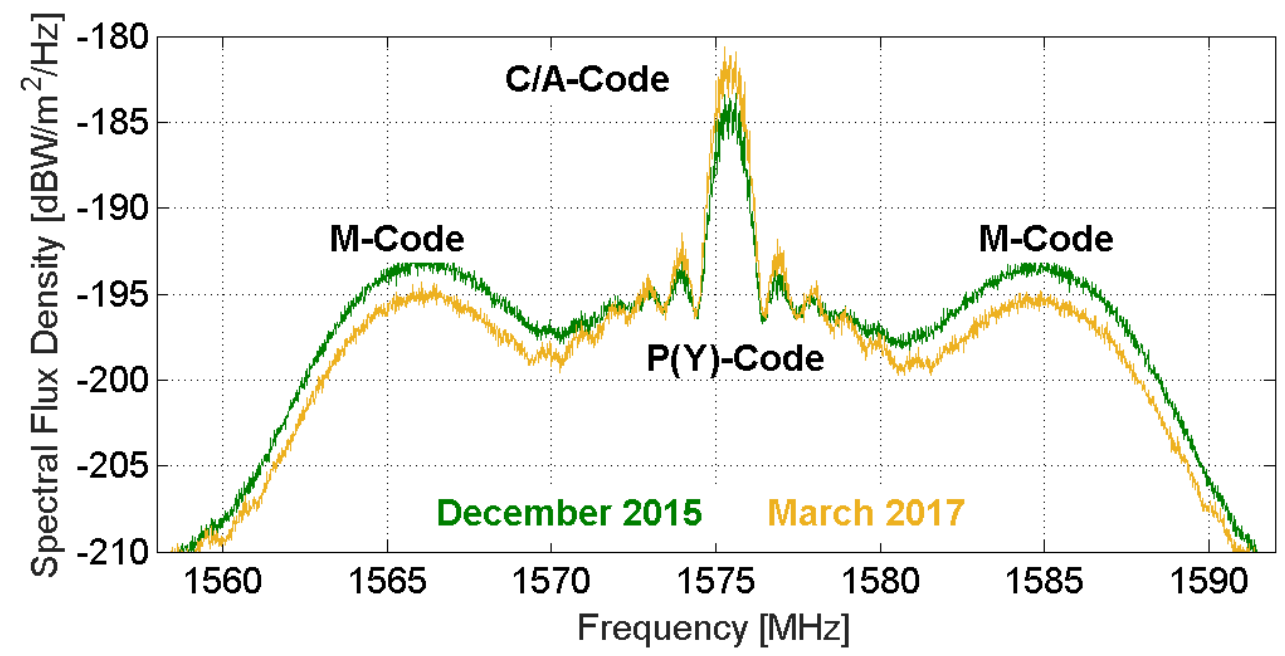

Figure 3: L1 spectral flux density of PRN 29 (SVN 57) for December 07, 2015 (normal C/A-code power level) and March 04, 2017 (increased C/A-code power level).

The analysis of the spectral flux density in the L1 band has confirmed the increase in the C/A-code power and also revealed a decrease in the M-code power, which could not be detected based on the geodetic GNSS receiver measurements. Further insight into whether the total radiated power in the L1 band has changed or remained constant can be gained from inspecting the equivalent isotropically radiated power (EIRP). Figure 4 depicts the EIRP for PRN 29 (SVN 57) measured on four different days. The first two measurements were taken on December 7, 2015 and October 16, 2016, prior to the reconfiguration of the satellite and are plotted in dark blue (cross) and green (diamond), respectively. The other two measurements were taken on March 4 and 23, 2017, after the reconfiguration. The plot shows that the total emitted power of the satellite has not increased since all three curves are roughly at the same level. Instead, the power has been redistributed among the different signal components. The small differences in the boresight-dependent variations are caused by the fact that the satellite's antenna gain pattern is not radially symmetric. 


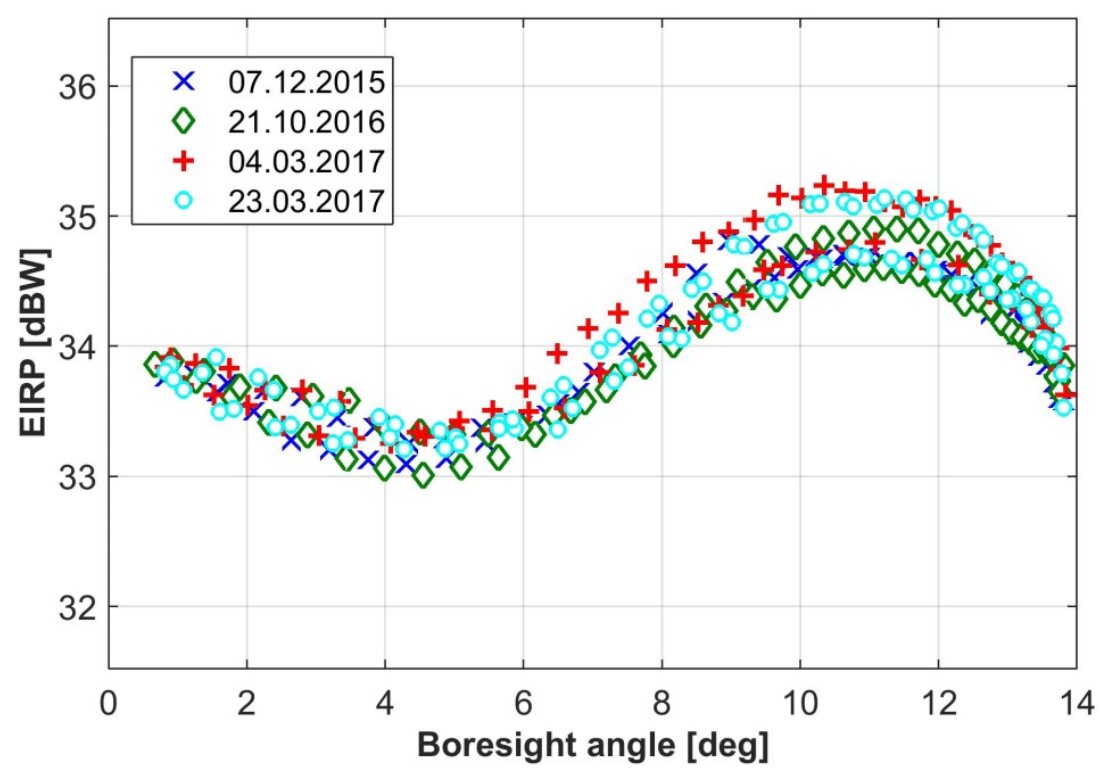

Figure 4: Equivalent isotropically radiated power (EIRP) over boresight angle for PRN 29 (SVN 57) measured on December 7, 2015 (cross), October 16, 2016 (diamond), March 4, 2017 (plus sign) and March 23, 2017 (circle).

\section{ANALYSIS OF TRANSMIT POWER FOR C/A-, P(Y)- AND M-CODE SIGNAL COMPONENTS}

In this paper, three different methods to determine the transmit power for each single signal component and the relation between the individual components are described. The main focus of the results section is given to the signal amplitude and power change and not the absolute power. But the methods could be finally also used to derive the absolute power of the signal and signal components.

The methods discussed within the next section are based on:

- Signal spectra

- $\quad$ In-phase and quadrature (IQ) -constellation diagram

- $\quad$ Transfer function of the satellite

After RF translation to baseband and low-pass filtering of the signal, either the signal spectra or the I and Q signal components were captured by a digitizer. Note that the measurement data regardless of spectra or I and Q samples are affected by the propagation path from the satellite to the measurement system and by the measurement system itself. For precise and accurate analysis results it is mandatory to compensate the received signal for all added effects during signal propagation and measurement.

The observables can expressed as

$\tilde{y}(t)=y(t)+\epsilon_{\text {atm }}(t)+\epsilon_{\text {meas }}(t)+n(t)$

with

$y(t): \quad$ Signal transmitted by the satellite

$\varepsilon_{a t m}(t):$ Atmospheric signal deformation

$\varepsilon_{\text {meas }}(t)$ : Measurement system behavior/deformation 
$n(t)$ Thermal noise

The terms $\varepsilon_{a t m}(t), \varepsilon_{\text {meas }}(t)$ and $n(t)$ modify the observed raw data so that it is not exactly the signal radiated by the satellite. Using a high gain antenna with a gain to system noise temperature (G/T) of approximately $28 \mathrm{~dB} / \mathrm{K}$ in the L-band, the noise can be neglected. Furthermore, it can be assumed that atmospheric (especially ionospheric) distortion in the central European region is small and might be negligible or it can be corrected by appropriate models such as total electron content (TEC) maps or estimation methods (Thoelert et al., 2017). With the precise knowledge of the measurement system behavior this part of the signal deformation of the recorded samples can also be corrected. Additionally, it is necessary, especially for the IQ-data, to remove the Doppler effect caused by the satellite movement. Finally, after solving the calibration issues regarding atmospheric effects and the measurement system influence, one can start with the signal analysis. Thus, it can be stated that the transmitted signal of the satellite is very close to the measured and calibrated signal.

Let the sampled signals $\mathrm{y}\left(\mathrm{nT}_{\mathrm{s}}\right)=\mathrm{y}[\mathrm{n}]$ and $\tilde{\mathrm{y}}\left(\mathrm{nT}_{\mathrm{s}}\right)=\tilde{\mathrm{y}}[\mathrm{n}]$ be represented by

$\mathbf{y}=\left[y[1], \ldots, y\left[N_{y}\right]\right]^{T} \in \mathbb{C}^{N_{y} \times 1}$

$\tilde{\mathbf{y}}=\left[\tilde{y}[1], \ldots, \tilde{y}\left[N_{y}\right]\right]^{T} \in \mathbb{C}^{N_{y} \times 1}$

where $T_{s}$ is the sampling duration and $f_{s}=1 / T_{s}$ is the sampling frequency. The discrete Fourier transform (DFT) of $\tilde{\mathbf{y}}$ can be given in matrix notation as

$\tilde{\mathbf{y}}_{\mathcal{F}}=\mathbf{W}_{N_{y}} \tilde{\mathbf{y}}$

where the $k, n$-th element of the DFT matrix $\mathbf{W}_{N_{y}}$ can be expressed as

$\left[\mathbf{W}_{N_{y}}\right]_{k, n}=e^{-j 2 \pi k n / N_{y}}$

An estimate of the power spectral density (PSD) or periodogram of $\tilde{\mathbf{y}}$ can be given as

$\tilde{\mathbf{y}}_{\mathcal{P}}=\frac{1}{N_{y}}\left(\tilde{\mathbf{y}}_{\mathcal{F}} \odot \tilde{\mathbf{y}}_{\mathcal{F}}^{*}\right)$

where $\odot$ denotes the Hadamard-Schur product (element-wise multiplication) and $*$ denotes complex conjugate.

\section{Spectrum}

The L1 band spectrum of the GPS IIR-M signal as shown in Figure 3 is the result of the superposition of the four signal components, which are the C/A-, P(Y)-, M-code and the so-called intermodulation product. The latter is a product of the three usable signals and is transmitted to reach constant envelope conditions, but does not contain usable information. The power allocation factors of the individual signal components can then be estimated through a fit of the PSD of the measurement signal $\tilde{y}$ to the computed PSD based on the theoretical signal. The undistorted interplex (or combined) signal comprised of $M$ signal components can be given as

$\mathbf{x}=\sum_{m=1}^{M} \beta_{m} \mathbf{x}_{m}$

where each signal component can be given as 
$\mathbf{x}_{m}=\left[x_{m}[1], \ldots, x_{m}\left[N_{y}\right]\right]^{\mathrm{T}} \in \mathbb{C}^{\mathrm{N}_{\mathrm{y}} \times 1}$

with $\forall_{m=1}^{M}\left\|\mathbf{x}_{m}\right\|_{2}^{2}=1$, superscript T denoting transpose, and $\|.\|_{2}^{2}$ denoting the square of the $l^{2}$ - norm . $\beta_{m}$ are the power allocation factors of the $M$ signal components and

$\boldsymbol{\beta}=\left[\beta_{1}, \ldots, \beta_{M}\right]^{T} \in \mathbb{R}^{M \times 1}$.

Assuming that the different signal components have small spectral overlap, the power allocation factors of the different signal components of the interplex signal can be estimated by solving the following least-squares problem

$\widehat{\boldsymbol{\beta}}=\operatorname{argmin}_{\boldsymbol{\beta}}\left\{\left\|\widetilde{\boldsymbol{y}}_{\mathcal{P}}-\mathbf{C}^{T} \widetilde{\boldsymbol{\beta}}\right\|_{2}^{2}\right\}$

where

$\mathbf{C}=\left[\begin{array}{c}\mathbf{x}_{\mathcal{P}}^{\mathrm{T}} \\ \vdots \\ \mathbf{x}_{\mathcal{P}}^{\mathrm{T}}\end{array}\right]$

and

$\widetilde{\boldsymbol{\beta}}=\left[\beta_{1}^{2}, \ldots, \beta_{\mathrm{M}}^{2}\right]^{\mathrm{T}} \in \mathbb{R}^{\mathrm{M} \times 1}$.

Considering that $\mathbf{C} \in \mathbb{R}^{\mathrm{M} \times \mathrm{N}_{\mathrm{y}}}$ we can express the estimation problem in closed form as:

$\widehat{\widetilde{\boldsymbol{\beta}}}=\left(\mathbf{C} \mathbf{C}^{\mathrm{T}}\right)^{-1} \mathbf{C} \tilde{\mathbf{y}}_{\mathcal{P}}$

An estimate of $\boldsymbol{\beta}$ can be derived by taking, element-wise, the square root of $\widehat{\widetilde{\boldsymbol{\beta}}}$.

The signal shape of each individual component in the frequency domain and the combined signal, which can be observed using a spectrum analyzer, are illustrated in Figure 5. 


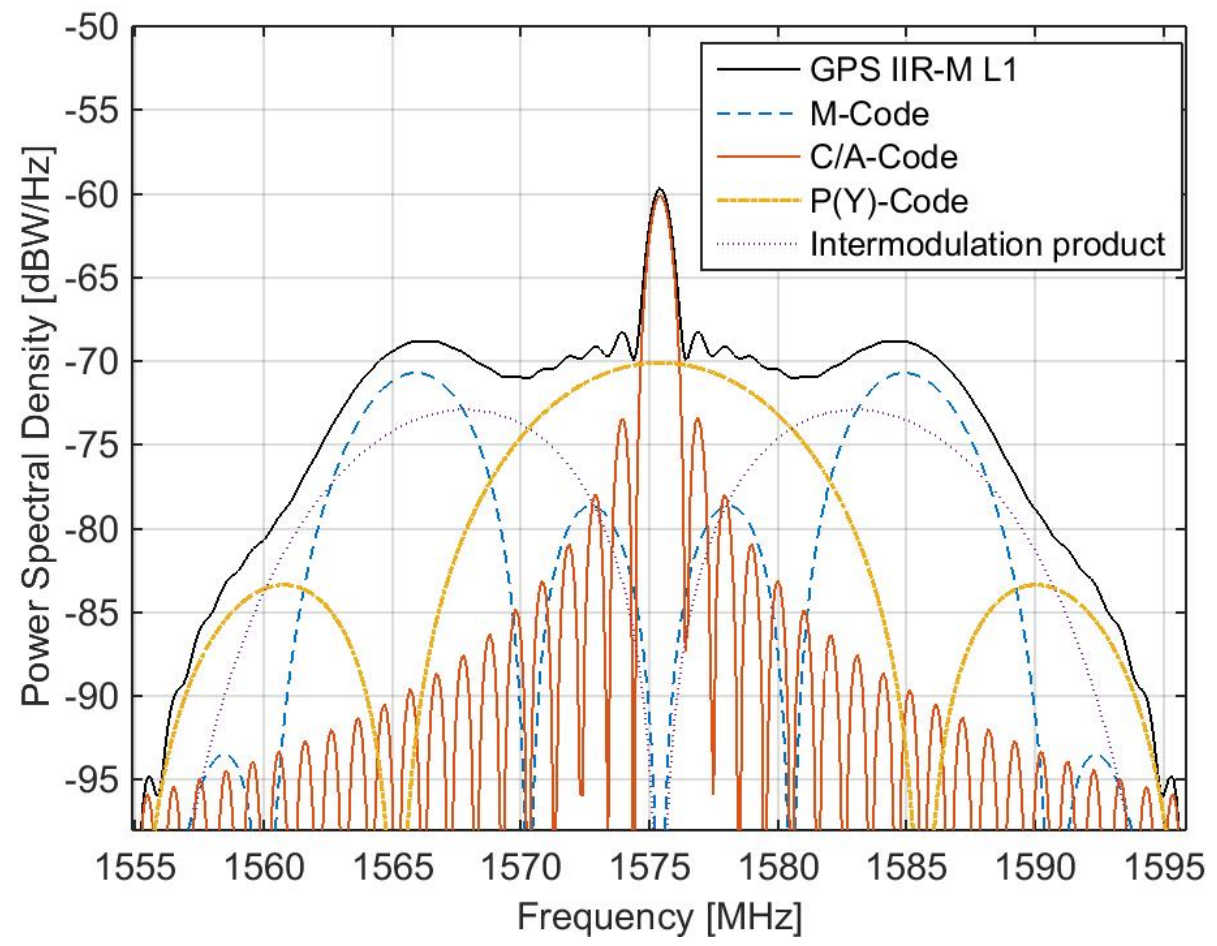

Figure 5: Theoretical power spectral density (PSD) envelope of GPS IIR-M L1 signal (solid), theoretical PSD of M-code (dashed), theoretical PSD of C/A-code (solid), theoretical PSD of P(Y)-code (dash-dotted) and theoretical PSD of the intermodulation product (dotted).

The results, using the least-squares estimation method described above, are presented in Table 3. The resulting spectra after best fit estimation over the frequency range of $1575.42 \mathrm{MHz} \pm 12 \mathrm{MHz}$ are represented for the data of December 2015 and March 2017 in Figure 6.

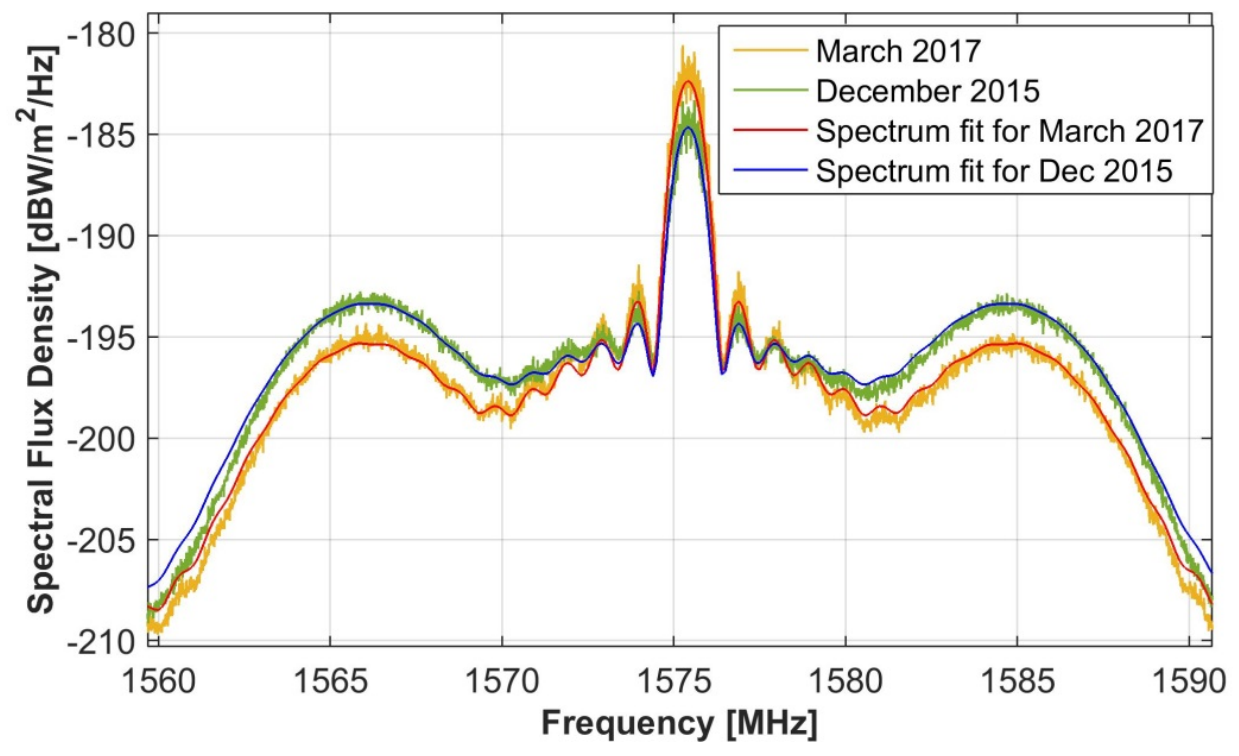

Figure 6: GPS IIR-M PRN 29 (SVN 57) L1 spectral flux density measurements and fits from December 2015 and March 2017. Solid lines are the least-squares fit curves. 


\section{IQ Constellation}

Partridge and Dafesh (2001) proposed a measurement methodology for determining the C/A-, P(Y)- and M-code signal power levels in on-orbit tests of the Block IIR-M and Block IIF GPS satellites. The aim of the method is to provide code power analysis without the aid of code tracking.

The basis of this method is a set of the already above-described calibrated and Doppler-shift-compensated I and Q samples of the satellite signal. The provided measurement samples can be used to produce an IQ constellation plot such as Figure 7. Using the I- and Q-states, the signal power of the modernized GPS signal of Block IIR-M and IIF satellites can be derived using the equations

$$
\begin{aligned}
& I_{2}=x_{p} C_{p}^{2}-x_{m} C_{m}^{2} \\
& Q_{2}=x_{c / a} C_{c / a}^{2}+x_{i m} C_{i m}^{2} \\
& I_{4}=x_{p} C_{p}^{4}-x_{m} C_{m}^{4} \\
& Q_{4}=x_{c / a} C_{c / a}^{4}+x_{i m} C_{i m}^{4}
\end{aligned}
$$

where $I_{i}$ and $Q_{i}$ are the measures from the IQ constellation plot within the corresponding plane and $x_{j}$ are the power allocation factors as given by

$$
x_{j}=\sqrt{2 P_{j}}, \quad j=\{\mathrm{C} / \mathrm{A}, \mathrm{P}(\mathrm{Y}), \mathrm{M}, \mathrm{IM}\}
$$

where $P_{j}$ is the power of the individual component and $C$ represent the set of constants of code and data values for each constellation data point as given in Table 2 where $j$ is the code and $i$ the constellation point.

Table 2: Code values $C$ multiplied by data $d(t)$, in-phase and quadrature values, and an $\left[I_{i}, Q_{i}\right]$ label for each constellation data point are listed according to Partridge and Dafesh (2001).

\begin{tabular}{|c|c|c|c|c|}
\hline $\mathbf{I}_{\mathbf{i}}, \mathbf{Q}_{\mathbf{i}}$ & $\mathbf{C}_{\mathbf{C} / \mathbf{A}}(\mathbf{t}) \cdot \mathbf{d}(\mathbf{t})$ & $\mathbf{C}_{\mathbf{P}(\mathbf{Y})}(\mathbf{t}) \cdot \mathbf{d}(\mathbf{t})$ & $\mathbf{C}_{\mathbf{M}}(\mathbf{t}) \cdot \mathbf{d}(\mathbf{t}) \cdot \sqrt{\mathbf{t}}$ & $\mathbf{C}_{\mathbf{I M}}(\mathbf{t})$ \\
\hline $\mathrm{I}_{1}, \mathrm{Q}_{1}$ & +1 & +1 & +1 & +1 \\
\hline $\mathrm{I}_{2}, \mathrm{Q}_{2}$ & +1 & +1 & -1 & -1 \\
\hline $\mathrm{I}_{3}, \mathrm{Q}_{3}$ & +1 & -1 & +1 & -1 \\
\hline $\mathrm{I}_{4}, \mathrm{Q}_{4}$ & +1 & -1 & -1 & +1 \\
\hline $\mathrm{I}_{5}, \mathrm{Q}_{5}$ & -1 & +1 & +1 & -1 \\
\hline $\mathrm{I}_{6}, \mathrm{Q}_{6}$ & -1 & +1 & -1 & +1 \\
\hline $\mathrm{I}_{7}, \mathrm{Q}_{7}$ & -1 & -1 & +1 & +1 \\
\hline $\mathrm{I}_{8}, \mathrm{Q}_{8}$ & -1 & -1 & -1 & -1 \\
\hline
\end{tabular}

Further details regarding this methodology including the mathematical derivation of the equations can be found in Partridge and Dafesh (2001).

In the following we apply the equations (14) - (18) to the gathered IQ data before February 7, 2017, and afterwards and solve them for the power allocation factors $x_{C / A}, x_{P(Y)}, x_{M}$ and $x_{I M}$. Figure 7 depicts the measured IQ constellation diagrams of GPS IIR-M-05 from October 21, 2016, before the power redistribution and from March 04, 2017, which represents the current status. Highlighted are the constellation points used for the calculation of the signal component power using the above described method of Partridge and Dafesh (2001). 

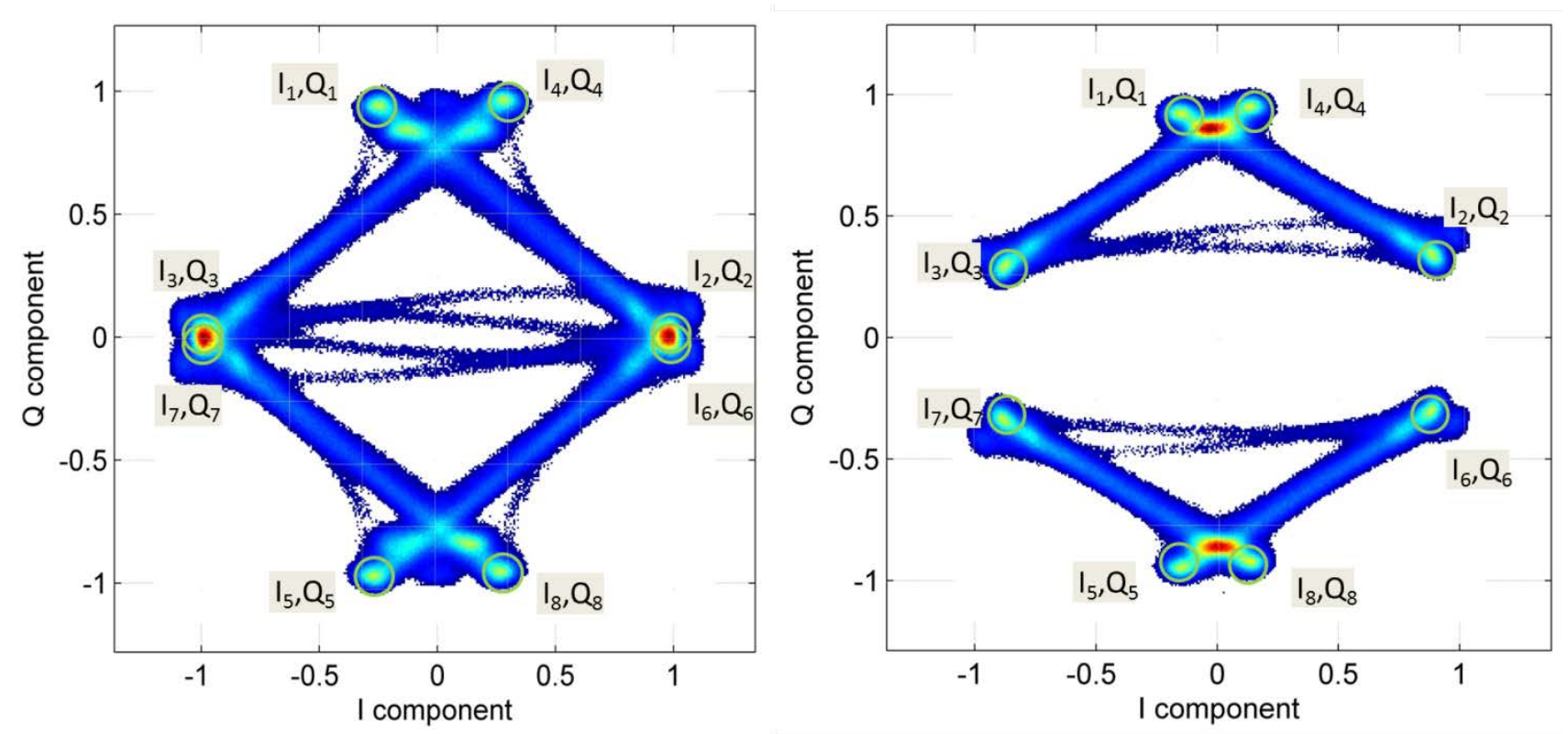

Figure 7: IQ constellation diagrams of GPS IIR-M PRN 29 (SVN 57) measured on October 21, 2016, (left) and on March 4, 2017 (right).

Table 3 shows the I- and Q-values corresponding to the constellation points $\left(I_{2}, Q_{2}, I_{4}, Q_{4}\right)$ needed for the computation of the power allocation factors $x_{j}$. This table also shows the results of the code power factor estimation and the variation after the modification for the four signal components of the GPS IIR-M satellite PRN 29 (SVN 57).

\section{Transfer Function}

A third method to determine the signal component power levels or the power allocation factors uses the same calibrated and Doppler-shift-compensated IQ-samples as input as the methods discussed before. This method is based on the transfer function estimation algorithm from Thoelert et al. (2014) and Vergara et al. (2016).

Let the impulse response of the transfer function of the satellite be given as

$\mathbf{h}=\left[h[1], \ldots, h\left[N_{h}\right]\right]^{\mathrm{T}} \in \mathbb{R}^{N_{h} \times 1}$

The digital distortions, which are present on each signal component, are measured in terms of chip time of each of the $M$ signal components of the signal $y(t)$ with $m=1, \ldots, M$. The digital distortion can be both positive and negative, and it cannot be larger in magnitude than one chip. Let us arrange the digital distortions present on the $M$ signal components in a vector:

$\boldsymbol{\eta}=\left[\eta_{1}, \ldots, \eta_{M}\right]^{\mathrm{T}}, \forall_{\eta_{m}} \in[-1,1]$

Hence, the ideal undistorted interplex signal is

$\mathbf{x}(\boldsymbol{\eta}, \boldsymbol{\beta})=\left[x[1], \ldots, x\left[N_{y}\right]\right]^{\mathrm{T}} \in \mathbb{C}^{\mathrm{N}_{\mathrm{y}} \times 1}$ 
and the convolution matrix with respect to $\mathbf{x}(\boldsymbol{\eta}, \boldsymbol{\beta})$ is denoted by $\mathbf{X}(\boldsymbol{\eta}, \boldsymbol{\beta}) \in \mathbb{C}^{N_{y} \times N_{h}}$ for $N_{y} \gg N_{h}$. The interplex signal is comprised of $M$ signal components and can be expressed as

$\mathbf{x}(\boldsymbol{\eta}, \boldsymbol{\beta})=\sum_{m=1}^{M} \beta_{m} \mathbf{x}_{m}\left(\eta_{m}\right)$

with $\forall_{m=1}^{M}\left\|\mathbf{x}_{m}\left(\eta_{m}\right)\right\|_{2}^{2}=1, \quad \mathbf{x}_{m}\left(\eta_{m}\right) \in \mathbb{C}^{N_{y} \times N_{h}}$ denoting the convolution matrix with respect to $\mathbf{x}_{m}\left(\eta_{m}\right)$, and

$\mathbf{x}_{m}\left(\eta_{m}\right)=\left[x_{m}[1], \ldots, x_{m}\left[N_{y}\right]\right]^{\mathrm{T}} \in \mathbb{C}^{\mathrm{N}_{\mathrm{y}} \times 1}$

In terms of convolution matrices, we can write

$\mathbf{X}(\boldsymbol{\eta}, \boldsymbol{\beta})=\sum_{m=1}^{M} \beta_{m} \mathbf{X}_{m}\left(\eta_{m}\right)$

The estimation problem of estimating the digital distortions, the power allocation factors, and the impulse response of the transfer function can be formulated as

$(\widehat{\boldsymbol{\eta}}, \widehat{\boldsymbol{\beta}}, \hat{\mathbf{h}})=\operatorname{argmin}_{\boldsymbol{\eta}, \boldsymbol{\beta}, \boldsymbol{h}}\left\{\|\tilde{\mathbf{y}}-\mathbf{X}(\boldsymbol{\eta}, \boldsymbol{\beta}) \mathbf{h}\|_{2}^{2}\right\}$.

Based on the measured and calibrated data, the chip sequences of the in-phase and quadrature signal components, as well as data symbols, can be detected. Note that the estimation of the sign of the digital distortion can be performed only if the secondary spreading codes (in the case of a pilot signal) or the preamble of the navigation message (in the case of a data-modulated ranging signal) are known. Without this information, the sign ambiguity cannot be solved. Doing so, the above-mentioned minimization problem in (25) can be solved by an iterative approach.

Differentiating the argument of (25) with respect to $\mathbf{h}^{\mathrm{H}}$ where superscript $\mathrm{H}$ denotes complex conjugate transposed, equating to zero, substituting into the argument of (25), and considering an estimate of the power allocation factors $\boldsymbol{\beta}$ of the $k-1$-th iteration we can write

$\widehat{\boldsymbol{\eta}}^{(k)}=\operatorname{argmin}_{\boldsymbol{\eta}}\left\{\widetilde{\mathbf{y}}^{\mathrm{H}} \mathbf{X}\left(\boldsymbol{\eta}, \widehat{\boldsymbol{\beta}}^{(k-1)}\right)\left(\mathbf{X}^{\mathrm{H}}\left(\boldsymbol{\eta}, \widehat{\boldsymbol{\beta}}^{(k-1)}\right) \mathbf{X}\left(\boldsymbol{\eta}, \widehat{\boldsymbol{\beta}}^{(k-1)}\right)\right)^{-1} \mathbf{X}^{\mathrm{H}}\left(\boldsymbol{\eta}, \widehat{\boldsymbol{\beta}}^{(k-1)}\right) \tilde{\mathbf{y}}\right\}$

Based on the determined digital distortions, then the estimate of the transfer function can be given as

$\hat{\mathbf{h}}^{(k)}=\left(\mathbf{X}^{\mathrm{H}}\left(\widehat{\boldsymbol{\eta}}^{(k)}, \widehat{\boldsymbol{\beta}}^{(k-1)}\right) \mathbf{X}\left(\widehat{\boldsymbol{\eta}}^{(k)}, \widehat{\boldsymbol{\beta}}^{(k-1)}\right)\right)^{-1} \mathbf{X}^{\mathrm{H}}\left(\widehat{\boldsymbol{\eta}}^{(k)}, \widehat{\boldsymbol{\beta}}^{(k-1)}\right) \tilde{\mathbf{y}}$

Finally, we can derive a new estimate of the power allocation factors

$\widehat{\boldsymbol{\beta}}^{(k)}=\left(\mathbf{G}^{\mathrm{H}}\left(\widehat{\boldsymbol{\eta}}^{(k)}, \hat{\mathbf{h}}^{(k)}\right) \mathbf{G}\left(\widehat{\boldsymbol{\eta}}^{(k)}, \hat{\mathbf{h}}^{(k)}\right)\right)^{-1} \mathbf{G}^{\mathrm{H}}\left(\widehat{\boldsymbol{\eta}}^{(k)}, \hat{\mathbf{h}}^{(k)}\right) \tilde{\mathbf{y}}$

where 
$\mathbf{G}\left(\widehat{\boldsymbol{\eta}}^{(k)}, \hat{\mathbf{h}}^{(k)}\right)=\left[\begin{array}{c}\left(\boldsymbol{X}_{1}\left(\hat{\eta}_{1}^{(k)}\right) \hat{\mathbf{h}}^{(k)}\right)^{\mathrm{T}} \\ \vdots \\ \left(\boldsymbol{X}_{M}\left(\hat{\eta}_{M}^{(k)}\right) \hat{\mathbf{h}}^{(k)}\right)^{\mathrm{T}}\end{array}\right]^{\mathrm{T}}$

The estimation of the parameters given in (26), (27), and (28) is iterated until convergence of the parameter estimates is achieved. The results for the GPS IIR-M satellite PRN 29 (SVN 57) are shown in Table 3.

\section{COMPARISON OF RESULTS}

The power allocation factors $\boldsymbol{x}_{j}$ do not represent the absolute power of the signal components. They correspond to amplitude factors regarding an input signal where the I and Q constellation is normalized to a unit circle. Absolute power could be calculated using these factors in combination with compensation of propagation loss, which is mainly the free space loss and knowledge about the satellite antenna pattern. However, the aim of this paper is to show the power redistribution between the individual signal components.

It is obvious from the results that all three considered methods provide the same result for C/A-code and slightly different results regarding the other signal components. This finding leads to a discussion about possible reasons and error sources regarding the used methods.

The method using the spectra has the advantage that you can average over the long measurement period and minimize the effect of temporary interferences or multipath. The main error source within this method is in the occurrence of signal distortions, possibly resulting in an asymmetric shape of the spectra, which can be seen in Figure 6 between the theoretical PSD curve and the related measurement curve. Using ideal PSDs may not provide the capability of achieving an appropriate fit. Consequently, the method, using the spectra, may not be suitable in case of strongly deformed signals. Within the present case, the asymmetry of the measured spectra is only small and the method can be used.

The IQ method is also very helpful using long data sets, because of the positive effect of averaging. But effects like band limitation and signal deformations will distort the constellation and thus it could result in a widened or stretched constellation point and such a deformation could result in choosing wrong estimates for the subsequent calculation.

The method based on the satellite transfer function has the highest complexity and prior estimation of the satellite payload characteristics but is nearly perfectly adapted to any signal distortion like band limitations, filtering or signal deformations. Therefore, this method should provide the most accurate results. But it must be kept in mind that the computational effort, especially for long data recordings, will be enormous.

The result for the C/A-code for all presented methods is a $2.37 \mathrm{~dB}$ change within the signal power. The narrow band spectrum is only affected by small distortions and the influence of nominal signal distortion (overshoot, ringing, etc.) is small related to the long chip duration. Therefore, neither the spectra nor the IQ constellation is significantly deformed and consequently, the results should be very close to the real value. The method using the transfer function should model all relevant signal distortions and therefore should be very precise in general. For the other signal components ( $\mathrm{P}(\mathrm{Y})$ - and $\mathrm{M}$-code) the influence of signal deformation become more relevant since band limitation and signal distortions more strongly affect these signals due to the shorter chip length.

A positive side effect of the power redistribution is the increase in the effective total power of the three usable signals based on the reduced power of the intermodulation product. If the power allocation factors of the C/A-, $\mathrm{P}(\mathrm{Y})$ - and M-code before and after the redistribution are compared, the power of the three signals has been increased in total by approximately $9 \%$. 
Table 3: Results of the estimation of the power allocation factors and the relative power change of each individual signal component of the GPS L1 signal transmitted from GPS IIR-M satellite PRN 29 (SVN 57) using three different methods.

\begin{tabular}{|c|c|c|c|c|c|c|}
\hline & \multicolumn{2}{|c|}{ IQ } & \multicolumn{2}{|c|}{ Correlation } & \multicolumn{2}{|c|}{ Spectrum } \\
\hline & Dec 2015 & Mar 2017 & Dec 2015 & Mar 2017 & Dec 2015 & Mar 2017 \\
\hline $\mathbf{I}_{2}$ & 0.999 & 0.946 & & & & \\
\hline $\mathbf{Q}_{2}$ & 0.016 & 0.320 & & & & \\
\hline $\mathbf{I}_{4}$ & 0.287 & 0.185 & & & & \\
\hline $\mathbf{Q}_{4}$ & 0.976 & 0.984 & & & & \\
\hline $\mathbf{x}_{\mathbf{C A}}$ & 0.4959 & 0.6516 & 0.5337 & 0.7012 & 0.5244 & 0.6892 \\
\hline $\mathbf{X}_{\mathbf{P}(\mathbf{Y})}$ & 0.3560 & 0.3808 & 0.4151 & 0.4216 & 0.3953 & 0.4082 \\
\hline $\mathbf{x}_{\mathbf{M}}$ & 0.6430 & 0.5654 & 0.5813 & 0.479 & 0.5701 & 0.4743 \\
\hline $\mathbf{x}_{\text {IM }}$ & 0.4797 & 0.3320 & 0.451 & 0.2863 & 0.4767 & 0.3227 \\
\hline$\Delta_{\mathrm{CA}}[\mathrm{dB}]$ & \multicolumn{2}{|c|}{2.37} & \multicolumn{2}{|c|}{2.37} & \multicolumn{2}{|c|}{2.37} \\
\hline$\Delta_{\mathbf{P}(\mathbf{Y})}[\mathrm{dB}]$ & \multicolumn{2}{|c|}{0.58} & \multicolumn{2}{|c|}{0.13} & \multicolumn{2}{|c|}{0.28} \\
\hline$\Delta_{\mathrm{M}}[\mathrm{dB}]$ & \multicolumn{2}{|c|}{-1.12} & \multicolumn{2}{|c|}{-1.68} & \multicolumn{2}{|c|}{-1.60} \\
\hline$\Delta_{\mathrm{IM}}[\mathrm{dB}]$ & \multicolumn{2}{|c|}{-3.20} & \multicolumn{2}{|c|}{-3.95} & \multicolumn{2}{|c|}{-3.39} \\
\hline
\end{tabular}

\section{SUMMARY AND CONCLUSIONS}

A rapid sequence of short satellite maintenance periods was carried out on all operational GPS Block IIR-M satellites between February 8 and 9, 2017. Carrier-to-noise-density ratio measurements from different geodetic receivers indicate an increase in the $\mathrm{L} 1 \mathrm{C} / \mathrm{A}$-code power of all satellites after the maintenance was performed. The measured $\mathrm{C} / \mathrm{N}_{0}$ is on average increased by approximately $1.5 \mathrm{~dB}$. Detailed analysis reveals that different types of receivers yield a slightly different increase for each satellite, most likely depending on the $\mathrm{C} / \mathrm{N}_{0}$ computation employed by the different receiver manufacturers. This scatter renders the observed carrier-to-noise-density ratio unusable for a precise estimation of the received signal power.

High-gain antenna measurements recorded before and after the maintenance have been used for a detailed analysis of the radiated power of the different signal components. Spectral flux density measurements confirm the increased power of the C/A-code signal on L1, but also show a slightly increased power of the P(Y)-code signal and a reduced power of the M-code. Measurements of EIRP confirm that the total radiated power has remained constant, though.

Three different methods have been applied to compute the transmit power increase or decrease of the three usable signal components as well as the losses through the intermodulation product. All three methods yield the same result for the increase in the $\mathrm{C} / \mathrm{A}$-code signal power of $2.37 \mathrm{~dB}$. In the case of the two military signals, the results vary. The increase for the $\mathrm{P}(\mathrm{Y})$-code ranges from $0.13 \mathrm{~dB}$ and $0.58 \mathrm{~dB}$ and the decrease of the M-code from 1.12 $\mathrm{dB}$ to $1.68 \mathrm{~dB}$. Most interesting is the significant reduction of losses through the intermodulation product, which shows a decrease in the power between $3.20 \mathrm{~dB}$ and $3.95 \mathrm{~dB}$ depending on the analysis method.

It can be summarized that the total available power onboard the spacecraft is used more efficiently for the transmission of navigation signals after the reconfigurations were performed in February 2017. It appears that these changes may be permanent on all Block IIR-M spacecraft. 


\section{ACKNOWLEDGEMENT}

Preliminary results have been published in the GPS World article "U.S. Air Force puts more power into GPS Block IIR-M C/A-code” in April 2017.

Richard B. Langley's GNSS research activity is primarily supported by the Natural Sciences and Engineering Research Council of Canada.

The authors want to thank the colleagues from the German Space Operations Center (GSOC) for supporting and operating the high gain antenna at DLR's ground station Weilheim, Germany.

This paper is a revised version of the paper in the proceedings of the ION GNSS+ 2017.

\section{REFERENCES}

Dow J.M., Neilan R.E. and Rizos C. (2009), The International GNSS Service in a changing landscape of Global Navigation Satellite Systems, Journal of Geodesy, 83(3-4):191-198, DOI; 10.1007/s00190-008-0300-3.

Ericson S., Shallberg K. and Edgar C. (2010), Characterization and Simulation of SVN49 (PRN01) elevation dependent measurement biases, Proc. ION ITM, pp. 963-974, San Diego, CA, USA.

Hartman T., Boyd L.R., Koster D., Rajan J.A. and Harvey J. (2000), Modernizing the GPS Block IIR Spacecraft, Proc. ION GNSS, pp. 2115-2121, Salt Lake City, UT, USA.

IS-GPS-200H (2014), Interface Specification IS-GPS-200: Navstar GPS Space Segment/Navigation User Segment Interfaces, Global Positioning Systems Directorate Systems Engineering \& Integration

Partridge M.D. and Dafesh P.A. (2001), Code Power Measurement Methodology for GPS Block IIR-M and IIF On-orbit Test Procedures., Proc. ION GPS, pp. 2764-2772, Salt Lake City, UT, USA.

Rajan J.A. and Tracy J.A. (2003), GPS IIR-M: Modernizing the Signal-In-Space, Proc. ION NTM, pp. 484-493, Anaheim, CA, USA.

Thoelert S., Vergara M., Sgammini M., Enneking C., Antreich F., Meurer M., Brocard D. and Rodriguez C. (2014), Characterization of Nominal Signal Distortions and Impact on Receiver Performance for GPS (IIF) L5 and Galileo (IOV) E1 /E5a Signals, Proc. ION GNSS, pp. 3113-3128, Tampa, FL, USA.

Thoelert S., Hoermann U., Antreich F. and Meurer M. (2017), Ionospheric Effects on High Gain Antenna GNSS Measurements TEC Estimation and Correction, Proc. ION GNSS+, Portland, OR, USA.

Vergara M., Sgammini M., Thoelert S., Enneking C., Zhu Y. and Antreich F. (2016) Tracking Error Modeling in Presence of Satellite Imperfections. Navigation - Journal of the Institute of Navigation 63(1):3-13, DOI: 10.1002/navi.129. 